\title{
Ste5 Membrane Localization Allows MAPK Pathway Signaling in trans Between Kinases on Separate Scaffold Molecules
}

\author{
Rachel E. Lamson, ${ }^{1}$ Matthew J. Winters, ${ }^{1}$ and Peter M. Pryciak ${ }^{1,2, *}$ \\ ${ }^{1}$ Department of Biochemistry and Molecular Pharmacology \\ University of Massachusetts Medical School, Worcester, MA 01605, USA \\ ${ }^{2}$ Lead Contact \\ * Correspondance: peter.pryciak@umassmed.edu
}




\section{SUMMARY}

The MAP kinase cascade is a ubiquitous eukaryotic signaling module that can be controlled by a diverse group of scaffold proteins. In budding yeast, activation of the mating MAP kinase cascade involves regulated membrane recruitment of the archetypal scaffold protein Ste5. This event promotes activation of the first kinase, but it also enhances subsequent signal propagation through the remainder of the cascade. By studying this latter effect, we find that membrane recruitment promotes signaling in trans between kinases on separate Ste5 molecules. First, trans signaling requires all Ste5 domains that mediate membrane recruitment, including both protein-binding and membrane-binding domains. Second, artificial membrane tethering of Ste5 can drive trans signaling, bypassing the need for native localization domains. Third, trans signaling can occur even if the first kinase does not bind the scaffold but instead is localized independently to the plasma membrane. Moreover, the trans signaling reaction allowed us to separate Ste5 into distinct functional domains, and then achieve normal regulation of signal output by tethering one domain to the membrane and stimulating membrane recruitment of the other. Overall, the results support a heterogeneous "ensemble" model of signaling in which scaffolds need not organize multiprotein complexes but instead can serve as binding sinks that co-concentrate enzymes and substrates at specific subcellular locales. These properties relax assembly constraints for scaffold proteins, increase regulatory flexibility, and can facilitate both natural evolution and artificial design of new signaling proteins and pathways.

KEYWORDS: signal transduction, kinase, MAPK, GPCR, scaffold protein, Ste5, Fus3, yeast, pheromone, plasma membrane 


\section{INTRODUCTION}

Most signal transduction pathways begin at the plasma membrane. In addition to the unique role of the plasma membrane as the interface between the cell exterior and interior, localization of signaling proteins to the plasma membrane has the potential to influence their reactions by altering their access to activators, substrates, and cofactors. In principle, such effects can emanate from increases in protein concentration as a result of colocalization [1, 2]. These issues are relevant in the mating pathway of budding yeast, a model system for investigating eukaryotic signaling mechanisms [3, 4]. In this pathway (Figure 1A), extracellular mating pheromones are detected by a transmembrane $\mathrm{G}$ protein-coupled receptor (GPCR), which triggers dissociation of a heterotrimeric $G$ protein $(G \alpha \beta \gamma)$. The liberated $G \beta \gamma$ dimer then activates a downstream MAP kinase (MAPK) cascade in a manner that requires a crucial intermediary, Ste5, a multi-domain scaffold protein with binding domains for $G \beta \gamma$, membrane phospholipids, and pathway kinases (Figure 1A-B). Signal transmission involves dramatic changes in subcellular localization: when $\mathrm{G} \beta \gamma$ is activated, it triggers plasma membrane recruitment of Ste5, which thereby mediates membrane localization of its associated kinases [5-8]. The proper membrane localization of Ste 5 depends on the concerted action of multiple binding motifs (Figure 1B), including a RING-H2 domain that interacts with $\mathrm{G} \beta \gamma$, a short membrane-binding peptide called the PM domain, and phospholipid-binding sequences within a larger $\mathrm{PH}$ domain [9-11]. Essential interactions with pathway kinases are mediated by two globular regions, the $\mathrm{PH}$ domain and the VWA domain (Figure 1B).

The binding and membrane recruitment of Ste 5 by activated $\mathrm{G} \beta \gamma$ has multiple effects on signaling through the downstream pathway. First, it helps initiate signaling by allowing membrane-bound Ste20 molecules to activate the first Ste5-associated kinase, the MAP kinase kinase kinase (MAPKKK) Ste11 (Figure 1A, step 1) [5, 12]. Second, it enhances the efficiency of signal propagation from Ste11 through the remainder of the kinase cascade (Figure 1A, steps 23). Specifically, if Ste11 is constitutively pre-activated by mutation (thus bypassing Ste20), pathway output remains low until cells are treated with pheromone or Ste 5 is artificially localized to the plasma membrane $[13,14]$. An analogous effect occurs in the mammalian Raf-MEK-ERK cascade, where membrane localization can convert low Raf activity into high pathway output [15]. This ability of membrane localization to stimulate steps in the middle of the pathway, rather than just at the top, can help prevent crosstalk from other pathways that use shared components $[13,16]$. It can also help shape the input-output properties of the pathway, by 
favoring a graded response to increasing levels of stimulus [14]. Yet the molecular mechanisms for these effects on signal propagation are only partly understood. As one contributor, membrane contact is thought to release the Ste5 VWA domain from inhibition by the $\mathrm{PH}$ domain (Figure 1C), and thus promote the final step in which the MAP kinase kinase (MAPKK) Ste7 activates the MAPK Fus3 [16]. The current study investigates whether membrane localization can also promote the previous step, in which Ste11 activates Ste7.

Although scaffold proteins are usually assumed to promote reactions between proteins bound to one scaffold molecule, previous observations suggest that the Ste11 $\rightarrow$ Ste7 activation step can occur with the two kinases bound to different molecules of Ste5 (Figure 1D). Namely, Ste5 mutants that cannot bind either Ste11 or Ste7, and hence are non-functional when expressed alone, will complement each other when co-expressed in the same cell $[17,18]$. This finding implies that Ste11 can phosphorylate Ste7 molecules on a separate scaffold, or "in trans". At the time, this phenomenon was hypothesized to reflect dimerization of Ste5 [17, 18]. Moreover, because trans signaling required the RING-H2 domain, it was suggested that this domain mediates dimerization. However, these studies were performed prior to any published knowledge about subcellular localization of Ste5 and the pathway kinases, or their $G \beta \gamma$ regulated membrane association. In retrospect, we wondered if trans signaling might be a more general consequence of membrane colocalization that could be broadly applicable to a variety of signaling proteins and pathways. Therefore, we revisited this phenomenon to better understand its mechanistic implications. Here, we report that trans signaling requires all membrane localization sequences, and that membrane localization is indeed both necessary and sufficient for trans signaling. Moreover, the capacity for trans signaling allows the distinct kinase-binding domains of Ste5 to be separated and localized independently, and yet the pathway remains controllable by stimulus-mediated membrane recruitment of either domain. By colocalizing and concentrating reactants in a reduced subcellular volume, membrane localization can allow Ste5 to enhance signal propagation without any individual scaffold molecules being fully occupied with kinases. These properties provide functional flexibility that may foster rapid evolution of scaffold proteins, and they have broad implications for membranelocalized signaling in diverse pathways. 


\section{RESULTS}

\section{Trans signaling requires membrane localization}

We used the original inter-allelic complementation assay $[17,18]$ to test which Ste5 domains were required for trans signaling. In particular, we sought to determine if trans signaling requires a unique dimerization motif or all sequences involved in membrane recruitment. We started with two Ste5 mutants that have defects in kinase binding (Figure 2A) [18, 19]. One mutant, Ste5I504T, harbors a mutation in the $\mathrm{PH}$ domain that disrupts binding to Ste11. Another mutant, Ste5-V763A S861P (aka "VASP" [20, 21]), harbors a mutation in the VWA domain that disrupts binding to Ste7. These were compared to mutants with defects in membrane recruitment [5, 911] (Figure 2A): $\triangle P M$ (missing the $\mathrm{N}$-terminal membrane-binding motif), $\triangle$ RING (missing the G $\beta \gamma$-binding RING-H2 domain), G $\beta \gamma^{*}$ (missing residues 152-173, which disrupts G $\beta \gamma$ binding but not other RING-H2 interactions), or $\mathrm{PH}^{*}$ (mutations R407S K411S in the PH domain, which disrupt membrane interaction). Whereas the two mutants with kinase-binding defects could complement each other, they could not be complemented by any of the four membrane localization mutants (Figures 2B, S1A). For further tests we incorporated the localization mutations into the Ste5-VASP mutant, and measured signaling output by assaying transcription and MAPK activation. By any assay, the localization mutations disrupted the ability of Ste5VASP to complement Ste5-I504T (Figures 2C-D, S1B). To counter the possibility that the localization sequences might have cryptic dimerization functions, we replaced the PM domain with an unrelated membrane-binding motif [10] (Figure 2A): the PH domain from PLC $\delta$ (in one or two copies). This replacement restored trans signaling (Figures 2C-D, S1B), arguing that the deficiency in the $\triangle \mathrm{PM}$ mutant can be explained by its localization defect. Therefore, trans signaling requires all localization sequences in Ste5, and hence the role of the RING-H2 domain is not unique but instead exemplifies a more general need for membrane localization. Notably, the quantitative assays indicated that trans signaling is an efficient reaction (rather than a rare event), as the pathway output was within $25-50 \%$ of that obtained with intact Ste5 (Figures $2 \mathrm{C}$, S1A,C).

The MAPK phosphorylation assays provided additional insights into which signaling steps were disrupted by the localization mutations. As described below, our analyses suggested that both the Ste20 $\rightarrow$ Ste11 and Ste11 $\rightarrow$ Ste7 steps were affected. When Ste5-VASP and Ste5I504T were coexpressed, pheromone stimulated phosphorylation of both Fus3 and its semiredundant paralog, Kss1 (Figure 2D, middle, lane 4). But when Ste5-VASP was expressed 
alone, pheromone could activate only Kss1 and not Fus3 (Figure 2D, top, lane 4). As reported previously [20,21], this behavior implies that Ste5-VASP is competent to mediate activation of Ste11 but cannot promote the subsequent reactions; consequently, the activated Ste11 can dissociate from Ste5 to weakly drive these reactions "off scaffold", leading to weak activation of Kss1 but not Fus3 (which requires the VWA domain [22]). This Kss1 phosphorylation provides a useful proxy for the successful activation of Ste11 by Ste5-VASP. Notably, it was disrupted by all four localization mutations (Figure 2D, top and middle, lanes 5-8) suggesting that they prevent Ste11 from being activated by Ste20 at the plasma membrane. In principle, this defect in Ste11 activation could suffice to explain their failure to complement Ste5-I504T. Thus, to address whether subsequent steps were also affected, we used a pre-activated form of Ste11, called Ste11-Asp3 (which contains Asp replacements at three activating phosphorylation sites) [12]. This Ste11-Asp3 mutant bypasses its upstream activator, Ste20, yet pathway output is still regulated by pheromone $[13,14]$.

The experiments with Ste11-Asp3 (Figures 2C, right, and 2D, bottom) revealed several noteworthy points, which ultimately suggest that membrane colocalization can promote the Ste11 $\rightarrow$ Ste7 reaction. First, in the absence of pheromone, elevated P-Kss1 levels confirmed the constitutive activity of Ste11-Asp3, while the low levels of P-Fus3 confirmed that this response still required pheromone stimulation (Figure 2D, bottom, lanes 1-2). Second, the Ste5I504T mutant alone could not mediate the pheromone-induced increase in transcription and $\mathrm{P}$ Fus3 (Figure 2C, right, and Figure 2D, bottom, lane 3). This indicates that the stimulatory effect of pheromone cannot be explained simply by relieving inhibition of the VWA domain (in the Ste5-I504T protein), which would have been expected if Ste7 were fully activated by Ste11Asp3 prior to pheromone addition. Third, the addition of Ste5-VASP restored activation of Fus3 and transcription, but this required that the membrane localization sequences were intact (Figure 2D, bottom, lanes 4-10). This behavior suggests a need to co-localize Ste5-I504T and Ste5-VASP molecules at the membrane, in order to increase the mutual proximity of Ste11Asp3 and Ste7, and thereby promote the MAPKKK $\rightarrow$ MAPKK reaction in trans (Figure 2E). In other words, these results show that the role of membrane localization in trans signaling cannot be explained solely by a need to activate Ste11 (which is bypassed by Ste11-Asp3) or by a need to de-repress the VWA domain (which should occur for Ste5-I504T in the absence of Ste5VASP), and instead they support the view that the Ste11 $\rightarrow$ Ste7 reaction is promoted by the co-recruitment of the two distinct scaffold molecules. 


\section{Membrane localization of kinase-binding domains permits trans signaling}

To pursue the implications of our findings further, we asked if membrane localization could be sufficient for trans signaling (Figure 3A). Thus, we used a form of Ste5 that is tethered to the plasma membrane by a C-terminal transmembrane domain (CTM), under control of an inducible promoter $\left(P_{G A L 1}\right)$. Previous studies showed that expression of this Ste5-CTM fusion protein could activate signaling and bypass the need for pheromone, receptor, and $G \beta \gamma$ [5]. Not surprisingly, variants of this Ste5-CTM fusion that harbored either of the kinase-binding mutations (I504T or VASP) were defective when expressed alone (Figure 3B). However, coexpression of the two membrane-tethered mutants restored signaling (Figures 3B), and with an efficiency similar to that described earlier when trans signaling was triggered by pheromone (i.e., within $30-50 \%$ of wild-type counterparts). There was no signaling output if either mutant lacked the CTM domain, indicating that both mutants must be membrane-tethered for trans signaling to occur (Figures 3B). Notably, G $\beta \gamma$ played no role here, because the strains lacked the gene for the $G \beta$ subunit (Ste4). Furthermore, the results were unchanged when the PM and RING-H2 domains were removed by deleting the Ste5 N-terminus ( $\Delta \mathrm{N}$, Figure $3 \mathrm{~B})$; thus, when the role of these domains in membrane localization were bypassed, so too were their roles in trans signaling. We conclude that interaction of G $\beta \gamma$ with the RING-H2 domain is not strictly required for trans signaling, and that their normal requirement can be explained by their role in membrane recruitment.

\section{Definition of minimal domains for trans signaling}

Next, we used the Ste5-CTM fusions to determine the minimal domains of Ste5 required for trans signaling. We reasoned that artificial membrane tethering should allow removal of all sequences needed only for membrane localization, and thus any remaining requirements would define regions critical for signaling between kinases. In addition, because the two complementing molecules perform distinct roles, we expected that each partner could be reduced to the minimum sequence needed to perform its individual role, free of other constraints that might be imposed when a single polypeptide performs all roles.

Indeed, using a series of truncations, we found that each partner could be trimmed to a region comprising a single structural domain (Figures $3 \mathrm{C}, \mathrm{S} 2 \mathrm{~A}$ ). The partner that provides the Ste11-binding role could be trimmed to a fragment corresponding to the $\mathrm{PH}$ domain (residues 370-590), and its counterpart could be trimmed to a fragment corresponding to the VWA domain (residues 560-820). In each case, some further truncation was tolerated but led to reduced 
function (e.g., when the PH domain C-terminus was trimmed to 560, and when the VWA domain was trimmed at its $\mathrm{N}$-terminus to 590 or at its C-terminus to 800 ). These partial reductions might reflect a simple need for linker sequence, such as to allow sufficient separation from the membrane or the CTM domain; indeed, using the original complementation assay between Ste5-1504T and Ste5-VASP (Figure S2B), the C-terminus of the PH domain could be truncated even further (to residue 531). Ultimately, robust signaling could be achieved by combining the two minimal fragments encoding the $\mathrm{PH}$ and VWA domains (Figure 3B). Further controls showed that both fragments must be membrane-tethered (Figure $3 \mathrm{~B}$ ); this finding is notable because the isolated VWA domain should be relieved of auto-inhibition [16], and yet its ability to transmit signal still required membrane localization. Other controls using monomeric YFP and mCherry to replace an N-terminal GFP moiety showed that the signaling results did not rely on potential dimerization tendencies of GFP (Figure S2C). Collectively, the results show that trans signaling can be induced by simultaneously targeting to the membrane two minimal signaling domains of Ste5 - the PH and VWA domains - that provide two distinct functions. In this context, other sequences were dispensable.

\section{Stimulus-induced regulation of individual functions}

The preceding results suggested to us that it should be possible to split the functions of Ste 5 in two, and then have only one function controlled by the pheromone stimulus. To address this possibility, we used the membrane-tethered domains defined in the previous section (PH-CTM or VWA-CTM), and then asked if pheromone could still control pathway output by regulating the remainder of Ste5 (i.e., lacking the excised domain). For these experiments, when removing the Ste5 $\mathrm{PH}$ domain we replaced it with a heterologous $\mathrm{PH}$ domain from mammalian PLC $\delta$ (i.e., Ste5 $\triangle \mathrm{PH}:: \mathrm{PLC})$, to compensate for any defects in membrane localization.

Initially, we confirmed that the mutants lacking individual domains (Ste5- $\Delta$ VWA and Ste5$\triangle \mathrm{PH}:: \mathrm{PLC}$ ) were able to complement each other, and that each partner required its localization sequences to remain intact (Figure 4A,B). Then, we tested each mutant for complementation by a membrane-tethered version of the excised domain (Figure 4C,D), and found that pathway output was still regulated by pheromone in each case (i.e., Ste5 $\triangle$ VWA + VWA-CTM or Ste5 $\triangle \mathrm{PH}:: \mathrm{PLC}+\mathrm{PH}-\mathrm{CTM})$. Because in either context only one partner contained a $\mathrm{G} \beta \gamma$-binding domain, the results imply that pheromone controlled the ability of that partner to functionally engage with its membrane-localized counterpart. Furthermore, each pheromone-regulated partner still required the remaining localization sequences (PM and RING) (Figure 4B). Thus, 
separation of Ste5 into distinct functional portions allows pathway regulation by pheromone and $\mathrm{G} \beta \gamma$ to be conferred by either portion individually, without having all portions regulated simultaneously. Given that the PH and VWA domains can function on different polypeptides, we also asked if their order within the same polypeptide could be interchanged. Indeed, the resulting domain-swapped form of Ste5 functioned similarly to wild-type Ste5 (Figure S3). This finding further illustrates the functional modularity of Ste5, and it places constraints on how any conformational changes that might be triggered by $\mathrm{G} \beta \gamma$ binding could be propagated throughout the rest of the polypeptide. Overall, these results reveal flexibility in the point of regulatory input, and they provide a setting in which it is possible to dissect distinct stimulus-regulated events.

\section{Trans signaling by a membrane-localized, scaffold-free kinase}

We next explored whether directly tethering one of the pathway kinases to the membrane could eliminate the need for a scaffold protein to localize it. For this we used a membrane-tethered form of Ste11 (Ste11-Cpr), which contains a C-terminal "CCaaX" motif that gets modified with lipophilic groups [10]. In the trans-signaling assay, we asked if this Ste11-Cpr fusion could substitute for Ste5-VASP (the partner that normally provides the Ste11-binding role) and thus complement Ste5-I504T (Figure 5A). Indeed, in a ste5-I504T strain, expression of Ste11-Cpr on its own was not sufficient to activate signaling, but it could do so when pheromone was added. This response required Ste11 to be membrane-tethered, as it was not observed if the key Cys residues in the CCaaX motif were mutated (Cpr-SS). Thus, in this context trans signaling occurred between membrane-bound Ste11 and scaffold-bound Ste7 (Figure 5A, right). Furthermore, we found that Ste11-Cpr could also activate signaling when co-expressed with membrane-tethered forms of the Ste5 VWA domain (Figures 5B, S4A). Both observations emphasize that trans signaling is not inherently dependent on specific inter-scaffold contacts.

We investigated two further methods to drive signaling by kinase co-localization. In one case we used a construct, Ste20[N]-Ste11[C], in which the C-terminal kinase domain from Ste11 is fused to localization sequences from the Ste20 N-terminus (Figure $5 \mathrm{C}$, right). This fusion was previously shown to increase signaling in wild-type and ste11 $\Delta$ cells [23, 24]. Here, we found that it also could mediate trans-signaling with Ste5-I504T (Figure 5C), which was disrupted by mutations in the localization sequences from Ste20 that bind Cdc42 (CRIB*) or the plasma membrane $\left(B R^{*}\right)$. Because the Ste11[C] fragment includes only the catalytic domain of Ste11, it is expected to be constitutively active due to being freed from its inhibitory N-terminus [12]; therefore, the finding that its trans-signaling activity still required membrane localization implies 
that signaling was stimulated by co-localizing the active kinase with its substrate. Its partner molecule, Ste5-1504T, also required intact localization sequences, and this was true regardless of which specific molecule it was paired with (Figures 5D, S4B).

In another case, we used the Ste20 N-terminus to localize the Ste5 VWA domain (Ste20[N]5VWA) (Figure 5E, right), and then combined this chimera with Ste5[N]-Ste11[C] [23], in which the Ste11 catalytic domain is fused to the N-terminus of Ste5 (containing the PM and RING-H2 domains). This combination also yielded trans signaling, which again relied on localization sequences in each partner (Figure 5E). This result is significant because the two chimeric proteins should constitutively achieve two distinct activation steps that are normally controlled by pheromone (i.e., de-repression of both the Ste11 kinase domain and the Ste5 VWA domain), and yet signal output was still regulated by pheromone and localization. There was a notable increase in basal signaling ( $16 \%$ of maximum), suggesting that these deregulations impose a cost of promiscuous signaling and reduced dynamic range. Basal signaling was lower $(<4 \%$ of maximum) in a related context (Figure 5F) in which membrane localization of the Ste5 VWA domain was not constitutive but instead remained pheromone-induced (using Ste $\triangle \triangle \mathrm{PH}:: \mathrm{PLC}$ ). Curiously, in this arrangement there was greater tolerance for deleting the PM domain from the Ste5[N]-Ste11[C] chimera; the reasons for this are not known, but previous work indicates that the PM domain helps increase net affinity for membrane-bound $G \beta \gamma$ and can become less essential when Ste5 levels are elevated [10], and so perhaps this reduced affinity can also become tolerated when other critical pathway steps are enhanced or accelerated (e.g., due to release from inhibition). Altogether, these findings reveal a remarkable variety of arrangements in which kinase colocalization allows trans signaling while still retaining stimulus-mediated regulation.

\section{No evidence for MAPK activation in trans.}

Finally, we asked if the Ste7 $\rightarrow$ Fus3 reaction might also be able to occur in trans. To address this question, we co-expressed two Ste7 mutants (Figure S4C): one that lacks kinase activity but retains Fus3 binding sequences (Ste7-R220; [25]) and one that retains kinase activity but cannot bind Fus3 (Ste7-ND; [26]). We observed no complementation between these forms. While less conclusive than a positive result, this finding might imply that Ste 5 can only promote the Ste7 $\rightarrow$ Fus3 reaction in cis. We sought to probe this issue further using mutations in the Ste5 VWA domain, which has two distinct functional surfaces (Figure S4D): one that binds Ste7 and one that induces Fus3 to become a good substrate for Ste7 [22]. Unfortunately, while 
mutations in either region can severely disrupt the reaction in vitro (by 100-1000x; [22]), we found their impact on signaling in vivo to be surprisingly mild $(<2 \mathrm{x})$ (Figure S4E), and hence unsuitable for complementation tests. Curiously, stronger defects emerged (Figure S4F) when signaling was initiated late in the pathway by a pseudo-active form of Ste7 (Ste7-EE), which was the only input source used in the prior in vitro studies [22]. This context dependence was unexpected, and it likely holds mechanistic implications that will be of interest to future studies.

\section{DISCUSSION}

Our findings illuminate how signal transmission can be stimulated by colocalization of signaling proteins to the plasma membrane. In particular, we report that membrane localization of Ste5 promotes signaling in trans between kinases that are bound to separate scaffold molecules. This conclusion is supported by the findings that trans signaling requires all localization sequences in Ste5, that direct membrane tethering of either Ste5 or a pathway kinase can suffice to promote trans signaling, and that trans signaling can be activated by a diverse array of methods that achieve kinase co-localization. Moreover, the trans signaling reaction allowed us to divide Ste5 into distinct functional domains, and to achieve normal regulation of signaling when either one was stimulated to colocalize with its membrane-tethered counterpart. The overall findings suggest that the scaffold mediates membrane colocalization of pathway kinases, which can enhance their signaling interactions by concentrating them in a reduced subcellular volume (Figure 6). This property can relax assembly constraints for scaffold proteins and increase regulatory flexibility, which has broad relevance to the function and evolution of signaling pathways.

It is relatively straightforward to envision how membrane recruitment of a cytoplasmic protein can promote its interaction with a membrane-localized partner (e.g., as in the initial Ste20 $\rightarrow$ Ste11 step). Yet membrane recruitment can also promote interactions between two cytoplasmic proteins, by strongly increasing their local concentrations [2]. In theory, translocation of proteins to a reduced volume of cytoplasm adjacent to the plasma membrane can raise local concentrations by 100 - to 1000 -fold [1,27], which can increase the number of complexes between two proteins when even a moderate fraction of each (e.g., 10\%) is colocalized. Scaffold proteins are themselves expected to enforce mutual proximity between their bound partners, but membrane colocalization could still be beneficial if scaffold molecules are not fully occupied. This is necessarily true for the mutant scaffolds used in this study, yet 
even in wild-type cells only a minority of Ste5 molecules ( 10-25\%) are bound to any given kinase $[7,28]$. Thus, because membrane localization allows Ste5 to enhance signal propagation without any individual scaffold molecules being fully occupied with kinases, it suggests a general scenario in which scaffolds need not assemble individual multiprotein complexes, but instead can promote signaling by serving as binding sinks that concentrate reactants in specific subcellular regions. This scenario loosens functional constraints on the scaffold and hence fits with models in which signaling is governed by heterogeneous protein "ensembles" [29], rather than by homogeneous, unitary complexes. This alternative view can help explain effects of membrane localization on signal output in previous studies [13, 14], as well as the remarkable ease with which signaling networks can be reconfigured [24].

In principle, even stronger concentration effects could be achieved by recruiting proteins into the smaller volume of a specialized microdomain, for which there is precedent in metazoan MAPK pathways and other signaling systems [30,31]. It is unclear if such domains exist in the yeast pheromone pathway, although in some reports Ste5 has appeared localized to membrane puncta $[8,11,32]$, the nature of which is currently unknown. Signaling reactions can also be promoted by the opposite of colocalization: namely, by excluding enzymes that catalyze the reverse reactions, such as phosphatases. In the pheromone pathway, phosphatases that inactivate the MAPK are not co-recruited with it to the membrane, such that the active MAPK is partitioned from its antagonists [7]. So far, however, comparable information is not available for phosphatases that reverse the upstream reactions.

It remains unknown whether the Ste11 $\rightarrow$ Ste7 reaction in normal cells occurs more frequently in trans or in cis. This is a challenging question to probe experimentally, because while it is easy to devise a context in which signaling must occur in trans (e.g., using the mutants in this study), it is very difficult to devise one in which signaling must occur in cis; hence, at present we cannot directly compare their efficiencies or even verify that the cis reaction occurs. In our assays, the reduction in pathway output when trans signaling was required (compared to cells with WT Ste5) might roughly estimate the fraction of signaling that normally occurs in cis, although more complex scenarios cannot be excluded. There is no evidence that a steric constraint forbids the cis reaction, and instead current data favor a flexible linkage between the kinase-binding domains of Ste5. Thus, we suspect that the relative frequency of cis versus trans reactions is most likely dictated by the kinase occupancy of Ste5 
and the local concentration achieved by membrane recruitment. This issue remains a worthwhile subject for future studies, including those involving computational approaches.

Trans signaling behavior was originally attributed to Ste5 dimerization, but our findings suggest that it can be explained more readily by membrane colocalization of multiple Ste5 molecules. Indeed, the need for all localization sequences in Ste5 indicates that the earlier observed role for the RING-H2 domain does not imply a unique dimerization function but instead exemplifies a more general need for membrane recruitment. To date there are no examples of Ste5 mutants whose signaling defects can be clearly attributed to a dimerization defect. Biophysical methods show that full-length Ste5 is predominantly monomeric in intact cells [7, 28], although co-immunoprecipitation can be detected in cell extracts [11, 33]. Twohybrid assays can detect self-interaction for a Ste5 fragment (residues 25-587), but this does not require the RING-H2 domain ([17]; M.J.W. and P.M.P., unpublished observations). The isolated RING-H2 domain does not show appreciable self-interaction when fully intact [10, 34], but it does when perturbed by partial truncation [10] or mutation of zinc-chelating cysteines (M.J.W. and P.M.P., unpublished observations). It is also relevant that dimerization might be expected to allow Ste5 localization defects to be compensated by a localization-competent partner, but instead we found that the kinase-binding mutants could not complement any of the localization mutants. With the benefit of hindsight and further advances in the field, we suggest the most parsimonious interpretation is that trans signaling does not necessarily require Ste5Ste5 contacts but instead results from the colocalization of multiple Ste5 molecules at the membrane. It remains possible, of course, that Ste5-Ste5 contacts could make such reactions even more efficient by further increasing local concentration beyond that achieved by colocalization alone.

MAPK pathways exist in all eukaryotes and have conserved functions. Despite its essential role in pheromone response, Ste5 is not conserved as strongly as its pathway kinases [35], and hence it likely emerged as an addition to a pre-existing pathway. Indeed, membrane localization of the homologous MAPK cascade in filamentous fungi depends on a scaffold protein (HAM-5) that is unrelated to Ste5 $[36,37]$. Moreover, scaffold proteins for MAPK pathways in metazoans (e.g., KSR, JIP-1, etc.) are not related to their fungal analogs or to each other [38], suggesting that this functional category has evolved independently multiple times. The ability to acquire a new scaffolding function might be assisted by the properties studied here. The stimulatory effects of membrane colocalization provide substantial flexibility in the mechanism of 
localization, and allow different steps to be driven by different scaffold molecules or even different proteins. Thus, localization could provide a simple way of gaining a new regulatory input, which could then foster evolution of more complex features such as specific binding interactions and allosteric changes $[2,35]$. These notions complement those arising from other studies on the yeast pheromone pathway, in which domain shuffling among pathway components can generate novel signaling behaviors and reveal the tolerance of the network to reconfiguration, both of which can facilitate evolution [23, 24].

Collectively, the findings reported here and in prior studies $[5,12,16]$ suggest that membrane recruitment separately promotes all three kinase activation steps in the pheromone pathway; i.e., activation of Ste11 by membrane-localized Ste20, trans-activation of Ste7 by Ste11, and de-repression of the Ste5 VWA domain to allow activation of Fus3 by Ste7. Moreover, pheromone can still control pathway output when some of these steps are activated constitutively; i.e., when Ste11 is pre-activated by mutation $[13,14]$, or when the Ste5 VWA domain is freed from its cis-inhibitory PH domain (this study), or even both (this study). Thus, this pathway exhibits elements of both fine-tuning and coarse functional flexibility. That is, the ability to control multiple steps simultaneously could help increase the dynamic range of response and reduce basal signaling, while also imparting tolerance of suboptimal parameters for individual reactions. Moreover, the ability to control pathway output even when some steps are constitutively active could foster the evolution of new pathways or new pathway stimuli, by allowing diversity in the regulated step [39, 40]. By analogy, design of synthetic signaling pathways [41,42] is likely to benefit from an evolutionary process in which simple initial circuits are gradually refined by incorporating additional control points. 


\section{METHODS}

\section{Yeast Strains and Growth Conditions}

Standard procedures were used for growth and genetic manipulation of yeast [43, 44]. Yeast strains were in W303 or S288C (derived from YPH499) backgrounds. Cells were grown at $30^{\circ} \mathrm{C}$ in yeast extract/peptone medium with $2 \%$ glucose (YPD), or in synthetic (SC) medium (lacking specific nutrients appropriate to select for plasmids) with $2 \%$ glucose or raffinose. Strains and plasmids are listed in Tables S1 and S2, respectively. Mutations at the native ste5 genomic locus (I504T and VASP) were introduced by a two-step allele replacement method [43] using plasmids pPP2870 and pPP2871.

\section{Mating and Pheromone Response Assays}

Patch mating assays were performed between test strains (MATa) and a partner strain (PT2 $\alpha$ ) using methods described previously [45]. To measure transcriptional responses, cells harboring an integrated FUS1-lacZ reporter were treated with $5 \mu \mathrm{M} \alpha$ factor for $2 \mathrm{hr}$. For signaling induced by $P_{G A L 1}$-regulated genes, cells growing in SC medium with raffinose were supplemented with $2 \%$ galactose, and incubated for $3 \mathrm{hr}$ (with or without $5 \mu \mathrm{M} \alpha$ factor); in some experiments,

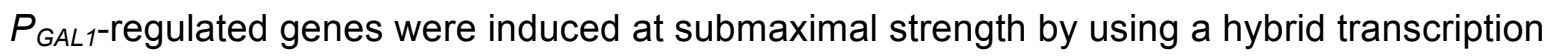
factor (Gal4 ${ }^{\mathrm{DBD}}$-hER-VP16, or "GEV") whose activity was controlled by the hormone $\beta$-estradiol [14]. Afterward, cells were collected and assayed for $\beta$-galactosidase activity by colorimetric assay as described previously [21]. To measure MAPK phosphorylation, cells were treated $\pm \alpha$ factor $(5 \mu \mathrm{M}, 15 \mathrm{~min}$ ) or, where applicable, treated first with galactose for $90 \mathrm{~min}$. and then incubated $\pm \alpha$ factor ( $5 \mu \mathrm{M}, 30 \mathrm{~min}$.). Afterward, 2-mL samples were harvested by centrifugation, then cell pellets were immediately frozen in liquid nitrogen and stored at $-80^{\circ} \mathrm{C}$.

\section{Cell Extracts and Immunoblotting}

Whole cell extracts were prepared by lysis in trichloroacetic acid as described previously [46], using frozen cell pellets from $2 \mathrm{~mL}$ cultures. Protein concentrations were measured by bicinchoninic acid (BCA) assay (Pierce \#23225), and equal amounts (10 $\mu \mathrm{g}$ ) were loaded per lane. Proteins were resolved by SDS-PAGE and transferred to polyvinylidene fluoride (PVDF) in a submerged tank. Primary antibodies were rabbit anti-phospho-p44/42 (1:1000, Cell Signaling Technology \#9101), rabbit anti-myc (1:200 Santa Cruz Biotechnologies \#sc-789), rabbit antiG6PDH (1:100000, Sigma \#A9521), or mouse anti-HA (1:1000, Covance \#MMS101R). HRPconjugated secondary antibodies were goat anti-rabbit (1:3000, Jackson ImmunoResearch 
\#111-035-144) or goat anti-mouse (1:3000, BioRad \#170-6516). Enhanced chemilluminescent detection used a BioRad Clarity substrate (\#170-5060). Exposures were captured on x-ray film.

\section{SUPPLEMENTAL INFORMATION}

Supplemental Information includes four figures and two tables.

\section{ACKNOWLEDGEMENTS}

This work was supported by a grant from the NIH (R01 GM057769) to P.M.P. We thank Beverly Errede, Wendell Lim, and Jeremy Thorner for sharing yeast plasmids, plus Alejandro ColmanLerner and Dan McCollum for feedback on the manuscript.

\section{AUTHOR CONTRIBUTIONS}

R.E.L. and M.J.W. performed the experiments; P.M.P. designed the project, analyzed the data, and wrote the paper.

\section{DECLARATION OF INTERESTS}

The authors declare no competing interests.

\section{REFERENCES}

1. Kholodenko, B.N., Hoek, J.B., and Westerhoff, H.V. (2000). Why cytoplasmic signalling proteins should be recruited to cell membranes. Trends Cell Biol 10, 173-178.

2. Kuriyan, J., and Eisenberg, D. (2007). The origin of protein interactions and allostery in colocalization. Nature 450, 983-990.

3. Bardwell, L. (2005). A walk-through of the yeast mating pheromone response pathway. Peptides 26, 339-350.

4. Alvaro, C.G., and Thorner, J. (2016). Heterotrimeric G Protein-coupled Receptor Signaling in Yeast Mating Pheromone Response. J Biol Chem 291, 7788-7795. 
5. Pryciak, P.M., and Huntress, F.A. (1998). Membrane recruitment of the kinase cascade scaffold protein Ste5 by the Gbetagamma complex underlies activation of the yeast pheromone response pathway. Genes Dev 12, 2684-2697.

6. van Drogen, F., Stucke, V.M., Jorritsma, G., and Peter, M. (2001). MAP kinase dynamics in response to pheromones in budding yeast. Nat Cell Biol 3, 1051-1059.

7. Maeder, C.I., Hink, M.A., Kinkhabwala, A., Mayr, R., Bastiaens, P.I., and Knop, M. (2007). Spatial regulation of Fus3 MAP kinase activity through a reaction-diffusion mechanism in yeast pheromone signalling. Nat Cell Biol 9, 1319-1326.

8. Bush, A., and Colman-Lerner, A. (2013). Quantitative measurement of protein relocalization in live cells. Biophys J 104, 727-736.

9. Mahanty, S.K., Wang, Y., Farley, F.W., and Elion, E.A. (1999). Nuclear shuttling of yeast scaffold Ste5 is required for its recruitment to the plasma membrane and activation of the mating MAPK cascade. Cell 98, 501-512.

10. Winters, M.J., Lamson, R.E., Nakanishi, H., Neiman, A.M., and Pryciak, P.M. (2005). A membrane binding domain in the ste 5 scaffold synergizes with gbetagamma binding to control localization and signaling in pheromone response. Mol Cell 20, 21-32.

11. Garrenton, L.S., Young, S.L., and Thorner, J. (2006). Function of the MAPK scaffold protein, Ste5, requires a cryptic PH domain. Genes Dev 20, 1946-1958.

12. Drogen, F., O'Rourke, S.M., Stucke, V.M., Jaquenoud, M., Neiman, A.M., and Peter, M. (2000). Phosphorylation of the MEKK Ste11p by the PAK-like kinase Ste20p is required for MAP kinase signaling in vivo. Curr Biol 10,630-639.

13. Lamson, R.E., Takahashi, S., Winters, M.J., and Pryciak, P.M. (2006). Dual role for membrane localization in yeast MAP kinase cascade activation and its contribution to signaling fidelity. Curr Biol 16, 618-623.

14. Takahashi, S., and Pryciak, P.M. (2008). Membrane localization of scaffold proteins promotes graded signaling in the yeast MAP kinase cascade. Curr Biol 18, 1184-1191.

15. Harding, A., Tian, T., Westbury, E., Frische, E., and Hancock, J.F. (2005). Subcellular localization determines MAP kinase signal output. Curr Biol 15, 869-873.

16. Zalatan, J.G., Coyle, S.M., Rajan, S., Sidhu, S.S., and Lim, W.A. (2012). Conformational control of the Ste5 scaffold protein insulates against MAP kinase misactivation. Science 337, 1218-1222.

17. Yablonski, D., Marbach, I., and Levitzki, A. (1996). Dimerization of Ste5, a mitogen-activated protein kinase cascade scaffold protein, is required for signal transduction. Proc Natl Acad Sci U S A 93, 13864-13869. 
18. Inouye, C., Dhillon, N., and Thorner, J. (1997). Ste5 RING-H2 domain: role in Ste4promoted oligomerization for yeast pheromone signaling. Science 278, 103-106.

19. Inouye, C., Dhillon, N., Durfee, T., Zambryski, P.C., and Thorner, J. (1997). Mutational analysis of STE5 in the yeast Saccharomyces cerevisiae: application of a differential interaction trap assay for examining protein-protein interactions. Genetics 147, 479-492.

20. Flatauer, L.J., Zadeh, S.F., and Bardwell, L. (2005). Mitogen-activated protein kinases with distinct requirements for Ste5 scaffolding influence signaling specificity in Saccharomyces cerevisiae. Mol Cell Biol 25, 1793-1803.

21. Winters, M.J., and Pryciak, P.M. (2018). Analysis of the thresholds for transcriptional activation by the yeast MAP kinases Fus3 and Kss1. Mol Biol Cell 29, 669-682.

22. Good, M., Tang, G., Singleton, J., Remenyi, A., and Lim, W.A. (2009). The Ste5 scaffold directs mating signaling by catalytically unlocking the Fus3 MAP kinase for activation. Cell 136, 1085-1097.

23. Peisajovich, S.G., Garbarino, J.E., Wei, P., and Lim, W.A. (2010). Rapid diversification of cell signaling phenotypes by modular domain recombination. Science 328, 368-372.

24. Sato, P.M., Yoganathan, K., Jung, J.H., and Peisajovich, S.G. (2014). The robustness of a signaling complex to domain rearrangements facilitates network evolution. PLoS Biol 12, e1002012.

25. Errede, B., Gartner, A., Zhou, Z., Nasmyth, K., and Ammerer, G. (1993). MAP kinase-related FUS3 from S. cerevisiae is activated by STE7 in vitro. Nature 362, 261-264.

26. Remenyi, A., Good, M.C., Bhattacharyya, R.P., and Lim, W.A. (2005). The role of docking interactions in mediating signaling input, output, and discrimination in the yeast MAPK network. Mol Cell 20, 951-962.

27. Yogurtcu, O.N., and Johnson, M.E. (2018). Cytosolic proteins can exploit membrane localization to trigger functional assembly. PLoS Comput Biol 14, e1006031.

28. Slaughter, B.D., Schwartz, J.W., and Li, R. (2007). Mapping dynamic protein interactions in MAP kinase signaling using live-cell fluorescence fluctuation spectroscopy and imaging. Proc Natl Acad Sci U S A 104, 20320-20325.

29. Mayer, B.J., Blinov, M.L., and Loew, L.M. (2009). Molecular machines or pleiomorphic ensembles: signaling complexes revisited. J Biol 8, 81.

30. Zhou, Y., and Hancock, J.F. (2015). Ras nanoclusters: Versatile lipid-based signaling platforms. Biochim Biophys Acta 1853, 841-849. 
31. Douglass, A.D., and Vale, R.D. (2005). Single-molecule microscopy reveals plasma membrane microdomains created by protein-protein networks that exclude or trap signaling molecules in T cells. Cell 121, 937-950.

32. Repetto, M.V., Winters, M.J., Bush, A., Reiter, W., Hollenstein, D.M., Ammerer, G., Pryciak, P.M., and Colman-Lerner, A. (2018). CDK and MAPK Synergistically Regulate Signaling Dynamics via a Shared Multi-site Phosphorylation Region on the Scaffold Protein Ste5. Mol Cell 69, 938-952 e936.

33. Feng, Y., Song, L.Y., Kincaid, E., Mahanty, S.K., and Elion, E.A. (1998). Functional binding between Gbeta and the LIM domain of Ste5 is required to activate the MEKK Ste11. Curr Biol 8, 267-278.

34. Walczak, M.J., Samatanga, B., van Drogen, F., Peter, M., Jelesarov, I., and Wider, G. (2014). The RING domain of the scaffold protein Ste5 adopts a molten globular character with high thermal and chemical stability. Angew Chem Int Ed Engl 53, 1320-1323.

35. Coyle, S.M., Flores, J., and Lim, W.A. (2013). Exploitation of latent allostery enables the evolution of new modes of MAP kinase regulation. Cell 154, 875-887.

36. Jonkers, W., Leeder, A.C., Ansong, C., Wang, Y., Yang, F., Starr, T.L., Camp, D.G., 2nd, Smith, R.D., and Glass, N.L. (2014). HAM-5 functions as a MAP kinase scaffold during cell fusion in Neurospora crassa. PLoS Genet 10, e1004783.

37. Serrano, A., Hammadeh, H.H., Herzog, S., Illgen, J., Schumann, M.R., Weichert, M., and Fleibetaner, A. (2017). The dynamics of signal complex formation mediating germling fusion in Neurospora crassa. Fungal Genet Biol 101, 31-33.

38. Morrison, D.K., and Davis, R.J. (2003). Regulation of MAP kinase signaling modules by scaffold proteins in mammals. Annu Rev Cell Dev Biol 19, 91-118.

39. Harrison, J.C., Zyla, T.R., Bardes, E.S., and Lew, D.J. (2004). Stress-specific activation mechanisms for the "cell integrity" MAPK pathway. J Biol Chem 279, 2616-2622.

40. Lee, J., Liu, L., and Levin, D.E. (2019). Stressing out or stressing in: intracellular pathways for SAPK activation. Curr Genet 65, 417-421.

41. Pryciak, P.M. (2009). Designing new cellular signaling pathways. Chem Biol 16, 249-254.

42. Lim, W.A. (2010). Designing customized cell signalling circuits. Nat Rev Mol Cell Biol 11, 393-403.

43. Rothstein, R. (1991). Targeting, disruption, replacement, and allele rescue: integrative DNA transformation in yeast. Methods Enzymol 194, 281-301.

44. Sherman, F. (2002). Getting started with yeast. Methods Enzymol 350, 3-41. 
45. Strickfaden, S.C., and Pryciak, P.M. (2008). Distinct roles for two Galpha-Gbeta interfaces in cell polarity control by a yeast heterotrimeric $\mathrm{G}$ protein. Mol Biol Cell 19, 181-197.

46. Pope, P.A., Bhaduri, S., and Pryciak, P.M. (2014). Regulation of cyclin-substrate docking by a G1 arrest signaling pathway and the Cdk inhibitor Far1. Curr Biol 24, 1390-1396.

47. Choi, K.Y., Satterberg, B., Lyons, D.M., and Elion, E.A. (1994). Ste5 tethers multiple protein kinases in the MAP kinase cascade required for mating in S. cerevisiae. Cell 78, 499-512.

48. Whiteway, M.S., Wu, C., Leeuw, T., Clark, K., Fourest-Lieuvin, A., Thomas, D.Y., and Leberer, E. (1995). Association of the yeast pheromone response $G$ protein beta gamma subunits with the MAP kinase scaffold Ste5p. Science 269, 1572-1575.

49. Bhattacharyya, R.P., Remenyi, A., Good, M.C., Bashor, C.J., Falick, A.M., and Lim, W.A. (2006). The Ste5 scaffold allosterically modulates signaling output of the yeast mating pathway. Science $311,822-826$.

50. Lamson, R.E., Winters, M.J., and Pryciak, P.M. (2002). Cdc42 regulation of kinase activity and signaling by the yeast p21-activated kinase Ste20. Mol Cell Biol 22, 2939-2951.

51. Takahashi, S., and Pryciak, P.M. (2007). Identification of novel membrane-binding domains in multiple yeast Cdc42 effectors. Mol Biol Cell 18, 4945-4956. 


\section{FIGURE LEGENDS}

FIGURE 1: Pheromone response pathway activation and role of the scaffold protein.

(A) The pheromone response pathway. Binding of pheromone to the GPCR triggers dissociation of the $G$ protein heterotrimer $(G \alpha \beta \gamma)$. The free $G \beta \gamma$ dimer then stimulates membrane recruitment of the scaffold protein, Ste5. A sequential cascade of three kinase activation steps ultimately activates the MAPK Fus3 (and its semi-redundant paralog, Kss1, not depicted), which induces downstream mating responses.

(B) Domain structure of Ste5, with binding targets of each region indicated above [10, 11, 19, 22, 47-49]. Note that the Fus3-binding motif ("D") allows Fus3 to trigger negative feedback but is not required for positive signaling [49]; instead, Fus3 must bind directly to Ste7 [26], as implied in panel $\mathrm{A}$.

(C) Lipid bilayer contact releases the Ste5 VWA domain from inhibition by the PH domain [16], allowing the VWA domain to promote the final Ste7 $\rightarrow$ Fus3 step by inducing Fus3 to be a receptive Ste7 substrate [22].

(D) Kinase activation can occur in trans, based on complementation between two Ste5 mutants that are each defective in binding one kinase $[17,18]$.

FIGURE 2: Trans signaling requires all membrane localization sequences in Ste5.

(A) Positions of Ste5 mutations that affect either membrane localization or kinase binding. In some experiments, the Ste5 PM domain is replaced with the $\mathrm{PH}$ domain from mammalian

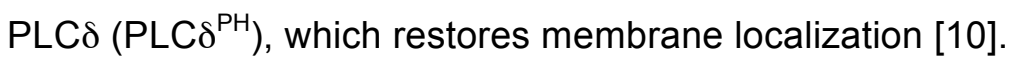

(B) Patch mating tests (shown in duplicate) of complementation between mutants with defects in kinase binding versus localization. Strains (PPY1974, PPY1975) with VASP or I504T mutations at the genomic locus (Ste5 \#1) harbored STE5-myc 13 $_{13}$ plasmids (Ste5 \#2). Also see Figure S1A.

(C) Transcriptional induction (FUS1-lacZ) in ste5-I504T cells (PPY1975), \pm STE11-Asp3, harboring STE5- $H A_{3}$ variants, treated with $\alpha$ factor $(5 \mu \mathrm{M}, 2 \mathrm{hr})$. Bars, mean $\pm \mathrm{SD}(\mathrm{n}=3)$.

(D) MAPK phosphorylation in strains (PPY2032, PPY1975) harboring STE5-HA $A_{3}$ variants \pm STE11-Asp3, treated $\pm \alpha$ factor $(5 \mu \mathrm{M}, 15 \mathrm{~min})$. Also see Figure S1B.

(E) Interpretation of findings. Ste11-Asp3 is depicted with a spiky outline to denote its preactivated state. In Ste5-I504T cells, Ste11-Asp3 yields only low Fus3 activation and pathway output, \pm pheromone stimulation (left panels). Adding Ste5-VASP allows signaling in trans, but only if membrane localization sequences are intact (right panels). 
FIGURE 3: Membrane-tethered Ste5 allows trans signaling and definition of minimal domains.

(A) Cartoons of normal Ste5 membrane recruitment (left) and trans signaling by membranetethered forms of Ste5 (center, right), as assayed in the next panels. For simplicity of illustration, the C-terminal transmembrane (CTM) domain is shown in a generic position.

(B) Trans signaling by Ste5-CTM derivatives bypasses requirement for $\mathrm{N}$-terminal PM and RING-H2 domains. FUS1-lacZ induction (mean \pm SD; $n=4$ ) was measured in ste4 $\Delta$ ste $5 \Delta$ cells (PPY886) coexpressing mutant variants of Ste5 or Ste $5 \Delta N$ (residues 215-917), with or without a CTM domain. $P_{\text {GAL1 }}$-driven constructs were induced with galactose $(3 \mathrm{hr})$.

(C) Minimal domains for trans signaling. Experiments were performed as in panel $\mathrm{B}$, using various truncations of Ste5-CTM. Red asterisks show positions of I504T and VASP mutations; numbers denote positions of new truncation endpoints. Bars, mean $\pm S D(n=3)$. Also see Figure S2B-D.

FIGURE 4: Stimulus-mediated regulation of individual Ste5 functions.

(A) Coexpression of Ste $5 \Delta V W A$ and Ste $5 \Delta \mathrm{PH}:$ :PLC (diagrammed at left) yields trans signaling, and both require intact PM and RING-H2 domains. FUS1-lacZ induction (mean \pm SD; $n=4$ ) was assayed $\pm \alpha$ factor (5 $\mu \mathrm{M}, 2$ hr.). Strain PPY2032 harbored $\mathrm{HA}_{3}$ - and myc $_{13}$-tagged variants of Ste $5 \Delta V W A$ and Ste $5 \Delta \mathrm{PH}:$ :PLC, respectively.

(B) Cartoon depiction of the signaling scenario in panel $A$.

(C) Combination of a stimulus-regulated partner (Ste5 5 VWA or Ste5 $\triangle \mathrm{PH}:: \mathrm{PLC}$ ) with an unregulated, membrane-tethered partner (VWA-CTM or PH-CTM) yields stimulus-regulated signaling. FUS1-lacZ induction (mean $\pm S D ; n=4$ ) was assayed after induction with galactose $\pm \alpha$ factor ( $5 \mu \mathrm{M}, 3 \mathrm{hr}$ ). Strain: PPY858. Also see Figure S3.

(D) Cartoon depictions of the signaling scenarios in panel C.

FIGURE 5: Trans signaling by membrane-tethered kinases and alternate recruitment methods.

(A) Complementation of the Ste5-I504T signaling defect by membrane-tethered Ste11 (Ste11Cpr). FUS1-lacZ induction (mean \pm SD; $\mathrm{n}=4$ ) was assayed in ste5-I504T cells (PPY1968) harboring STE5-myc 13 or $P_{\text {GAL1 }}$ STE11-Cpr plasmids, after induction with galactose $\pm \alpha$ factor $(5 \mu \mathrm{M}, 3 \mathrm{hr})$.

(B) FUS1-lacZ induction (mean $\pm \mathrm{SD} ; \mathrm{n}=4$ ) in cells (PPY2252) coexpressing the indicated

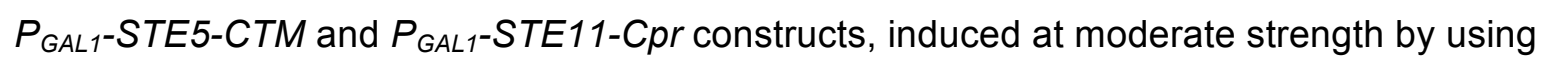
$10 \mathrm{nM} \beta$-estradiol (90 min). See also Figure S4A. 
(C) Ste5-I504T is complemented by Ste20[N]-Ste11[C], where the active Ste11 kinase domain is localized by the Ste20 N-terminus. FUS1-lacZ (mean $\pm S D ; n=4$ ) was assayed $\pm \alpha$ factor $(5 \mu \mathrm{M}, 2 \mathrm{hr})$. CRIB* and BR* denote mutations in Ste20 sequences that bind Cdc42 and plasma membrane, respectively [50, 51]. Strain: PPY2032.

(D) Localization sequences are required for Ste5-I504T to complement Ste5-VASP, Ste11-Cpr, or Ste20[N]-Ste11[C]. FUS1-lacZ induction (mean \pm SD) was assayed $\pm \alpha$ factor $(5 \mu \mathrm{M}, 2 \mathrm{hr}$; $\mathrm{n}=4$; PPY2032), or for Ste11-Cpr after induction with galactose $\pm \alpha$ factor $(5 \mu \mathrm{M}, 3 \mathrm{hr} ; \mathrm{n}=$ 3; PPY858). Also see Figure S4A.

(E) FUS1-lacZ induction (mean \pm SD; $\mathrm{n}=6$ ) in ste $5 \Delta$ cells (PPY2032) harboring plasmid-borne Ste5[N]-Ste11[C] and Ste20[N]-Ste5[VWA] variants, treated $\pm \alpha$ factor (5 $\mu \mathrm{M}, 2 \mathrm{hr})$.

(F) FUS1-lacZ induction (mean \pm SD; $n=6$ ) in ste5 $\Delta$ cells (PPY2032) harboring plasmid-borne Ste5[N]-Ste11[C] and Ste5 $\Delta \mathrm{PH}::$ PLC variants, treated $\pm \alpha$ factor $(5 \mu \mathrm{M}, 2 \mathrm{hr})$.

FIGURE 6: General model for concentrating kinases by membrane colocalization.

As the stimulus dose increases, an increasing fraction of molecules is redistributed to a reduced volume of cytoplasm adjacent to the plasma membrane. The increased concentration can favor protein-protein interactions and catalytic reactions among signaling proteins. Results in this study suggest that a scaffold protein can mediate this process by serving as a membranerecruited binding sink for distinct kinases that signal to each other without being bound to the same scaffold molecule. 
bioRxiv preprint doi: https://doi.org/10 1101/673855; this version posted June 17, 2019. The copyright holder for this preprint (which was not certified by peer review) is the author/funder, who has granted bioRxiv a license to display the preprint in perpetuity. It is made available under aCC-BY-NC 4.0 International license.

Figure 1

A

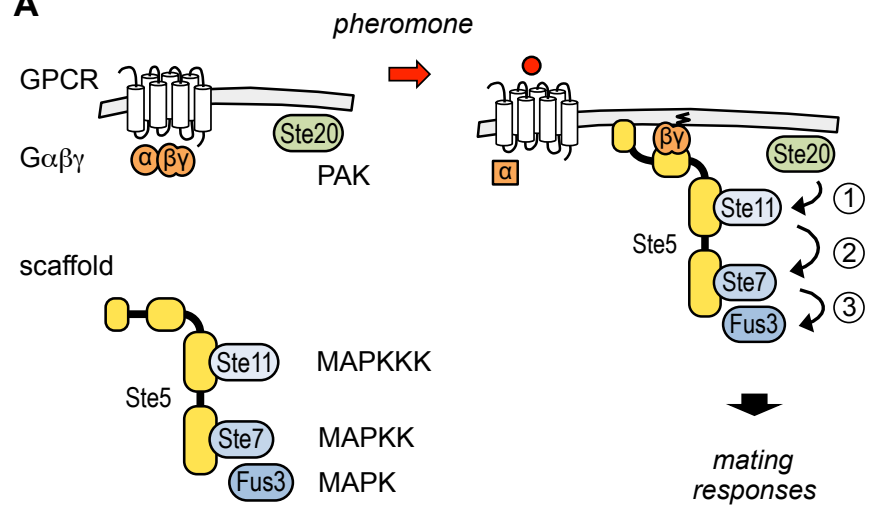

B

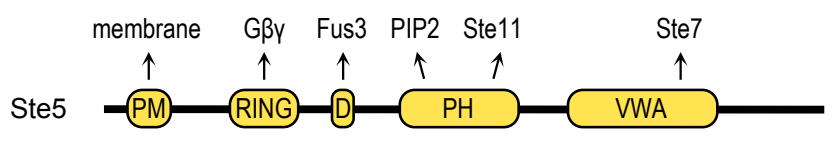

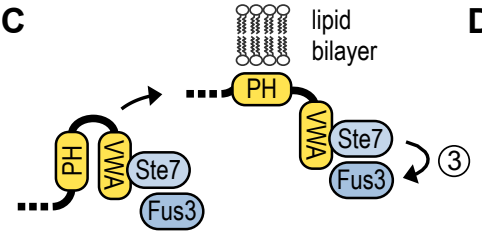

VWA domain de-inhibition

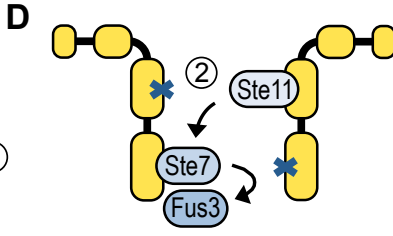

kinase activation in trans

FIGURE 1: Pheromone response pathway activation and role of the scaffold protein.

(A) The pheromone response pathway. Binding of pheromone to the GPCR triggers dissociation of the G protein heterotrimer $(G \alpha \beta \gamma)$. The free $G \beta \gamma$ dimer then stimulates membrane recruitment of the scaffold protein, Ste5. A sequential cascade of three kinase activation steps ultimately activates the MAPK Fus3 (and its semi-redundant paralog, Kss1, not depicted), which induces downstream mating responses.

(B) Domain structure of Ste5, with binding targets of each region indicated above [10, 11, 19, 22, 47-49]. Note that the Fus3-binding motif ("D") allows Fus3 to trigger negative feedback but is not required for positive signaling [49]; instead, Fus3 must bind directly to Ste7 [26], as implied in panel A.

(C) Lipid bilayer contact releases the Ste5 VWA domain from inhibition by the PH domain [16], allowing the VWA domain to promote the final Ste7 $\rightarrow$ Fus3 step by inducing Fus3 to be a receptive Ste7 substrate [22].

(D) Kinase activation can occur in trans, based on complementation between two Ste5 mutants that are each defective in binding one kinase $[17,18]$. 
bioRxiv preprint doi: https://doi.org/10.1101/673855; this version posted June 17, 2019. The copyright holder for this preprint (which was not certified by peer review) is the author/funder, who has granted bioRxiv a license to display the preprint in perpetuity. It is made available under aCC-BY-NC 4.0 International license.

Figure 2

A

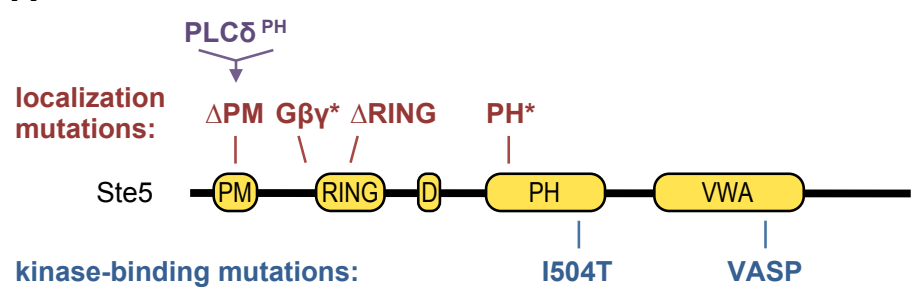

C

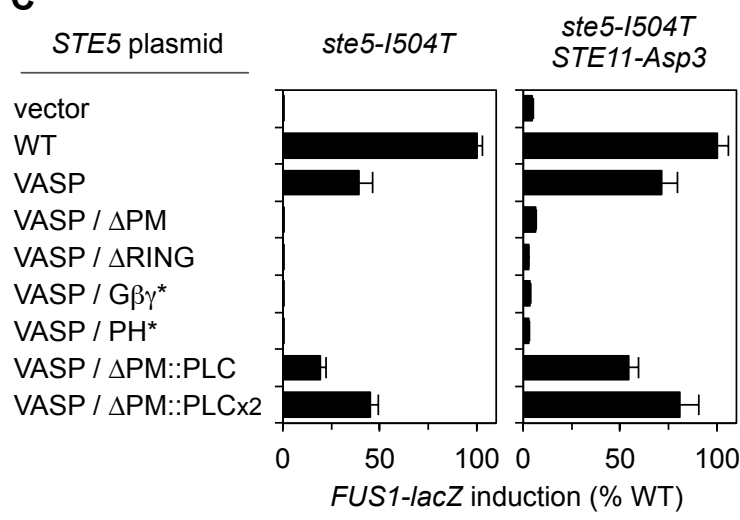

E

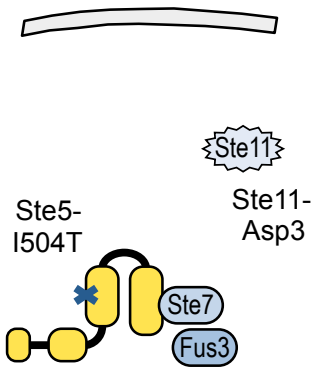

\section{+ pheromone}

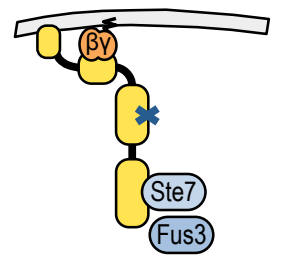

D

B

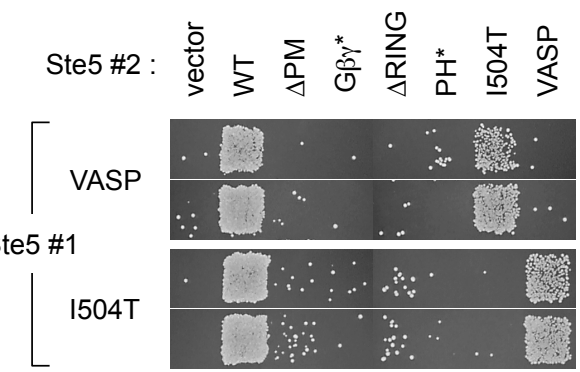

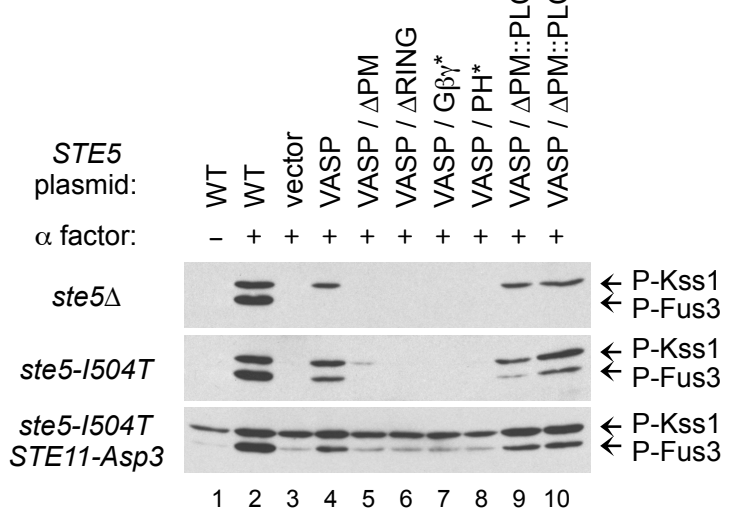


bioRxiv preprint doi: https://doi.org/10.1101/673855; this version posted June 17, 2019. The copyright holder for this preprint (which was not certified by peer review) is the author/funder, who has granted bioRxiv a license to display the preprint in perpetuity. It is made available under aCC-BY-NC 4.0 International license.

Figure 3

A G $\beta \gamma$-triggered
recruitment

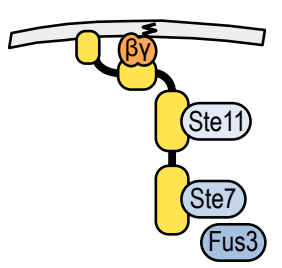

Ste $5 \Delta$ N-CTM: $1504 T+$ VASP

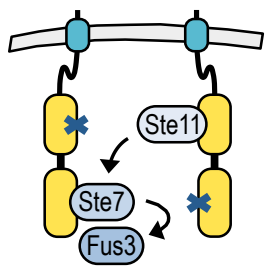

$\mathrm{PH}-\mathrm{CTM}+$ VWA-CTM

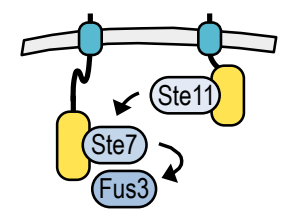

B

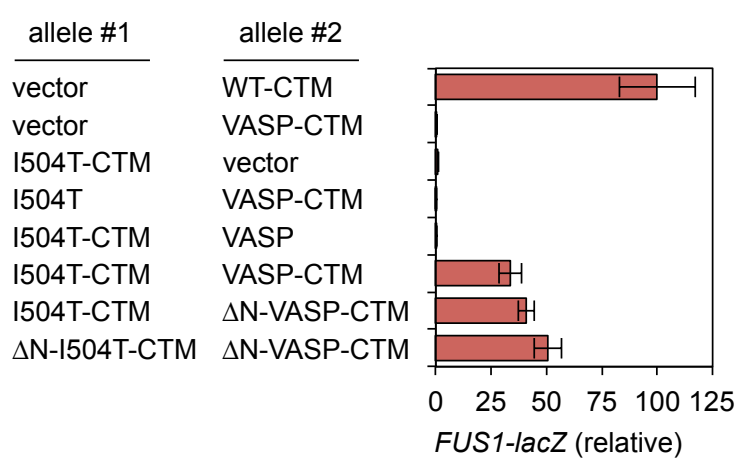

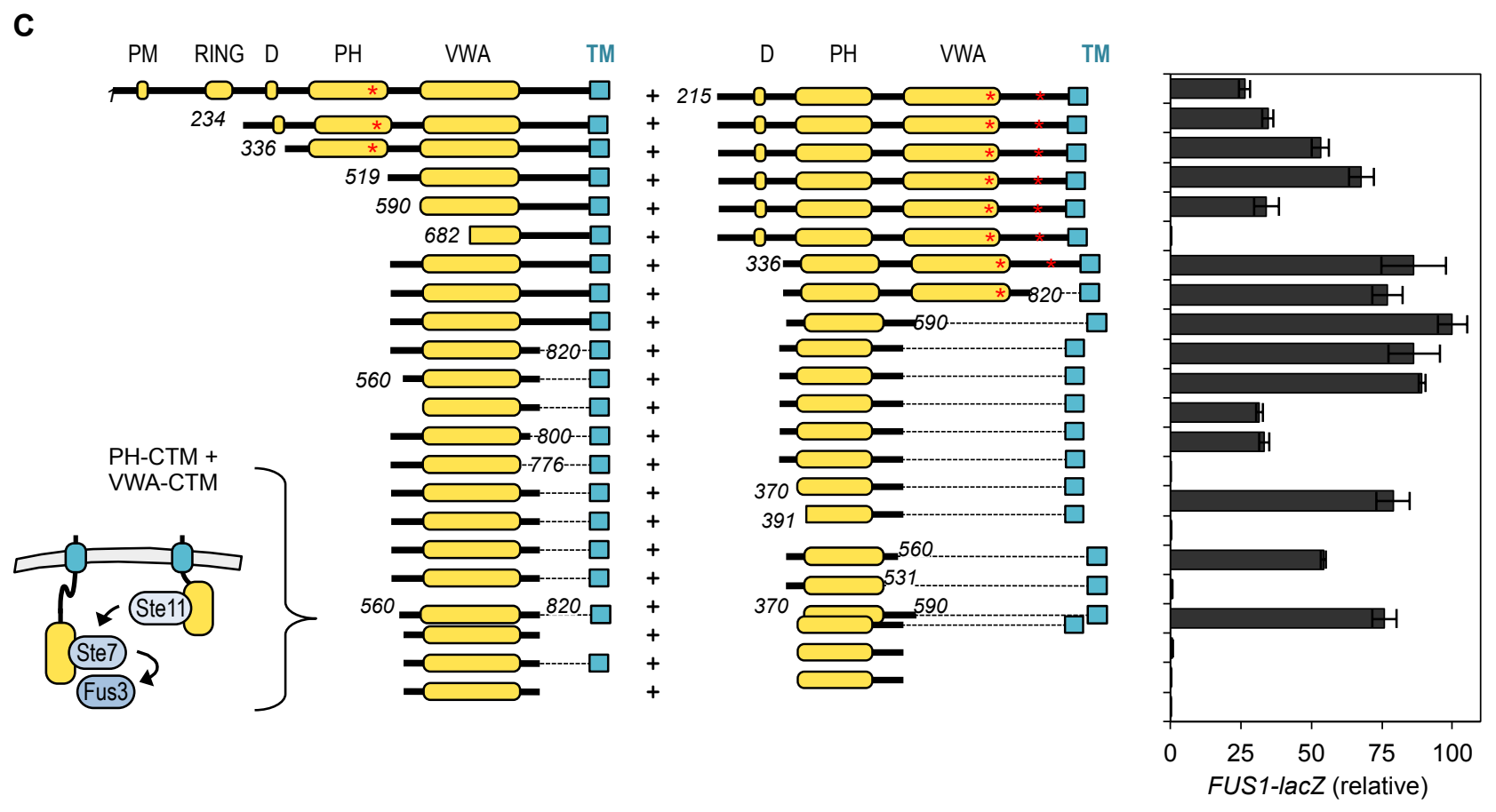

FIGURE 3: Membrane-tethered Ste5 allows trans signaling and definition of minimal domains.

(A) Cartoons of normal Ste5 membrane recruitment (left) and trans signaling by membrane-tethered forms of Ste5 (center, right), as assayed in the next panels. For simplicity of illustration, the C-terminal transmembrane (CTM) domain is shown in a generic position. (B) Trans signaling by Ste5-CTM derivatives bypasses requirement for N-terminal PM and RING-H2 domains. FUS1-lacZ induction (mean $\pm S D ; n=4$ ) was measured in ste4 $\Delta$ ste5 $\Delta$ cells (PPY886) coexpressing mutant variants of Ste5 or Ste5 $\Delta \mathrm{N}$ (residues 215-917), with or without a CTM domain. $P_{\text {GAL1 }}$-driven constructs were induced with galactose ( $3 \mathrm{hr}$ ).

(C) Minimal domains for trans signaling. Experiments were performed as in panel B, using various truncations of Ste5-CTM. Red asterisks show positions of I504T and VASP mutations; numbers denote positions of new truncation endpoints. Bars, mean \pm SD $(n=3)$. Also see Figure S2B-D. 
bioRxiv preprint doi: $\mathrm{https} / / \mathrm{doi}$ org/10.1101/673855; this version posted June 17,2019 . The copyright holder for this preprint (which was not certified by peer review) is the author/funder, who has granted bioRxiv a license to display the preprint in perpetuity. It is made available under aCC-BY-NC 4.0 International license.

Figure 4

A

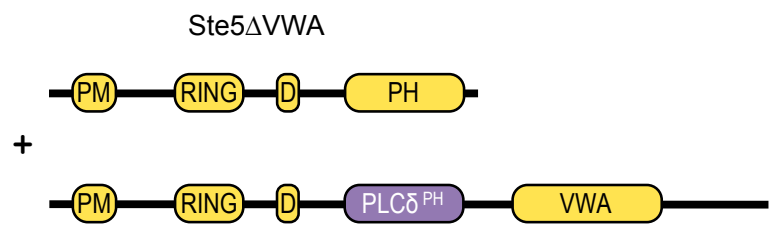

Ste5 $\triangle \mathrm{PH}:: \mathrm{PLC}$
Ste5 Ste5

$\Delta$ VWA $\Delta \mathrm{PH}:: \mathrm{PLC}$

none WT

WT WT

$\triangle P M \quad W T$

$\triangle \mathrm{RING} \quad \mathrm{WT}$

WT

WT

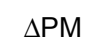

$\Delta \mathrm{RING}$

-

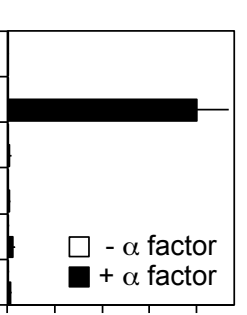

$0 \quad 255075100$

FUS1-lacZ (relative)
B Ste $5 \Delta \mathrm{VWA}+$ Ste $5 \triangle \mathrm{PH}:: \mathrm{PLC}$

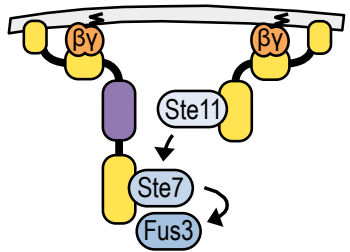

C

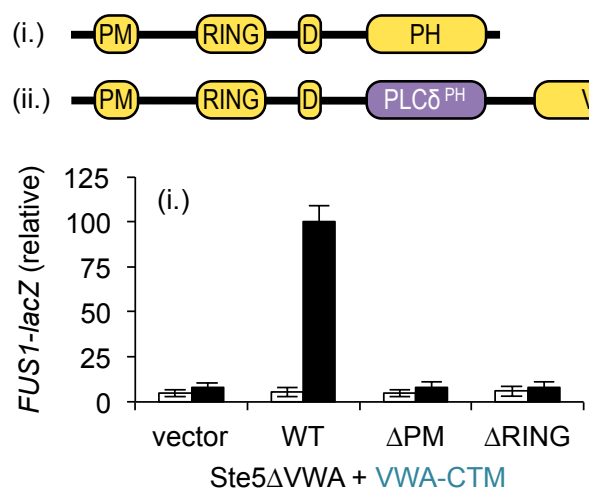

stimulus-regulated partner

Ste5 $\Delta$ VWA + VWA-CTM unregulated partner
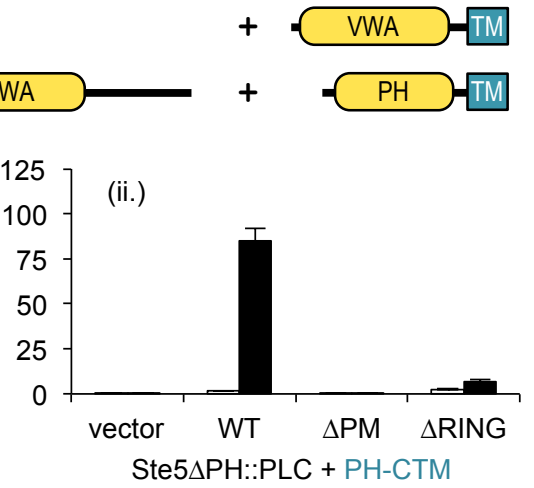

D

(i.)

(ii.)
Ste5 $\triangle \mathrm{VWA}+\quad$ Ste5 $\triangle \mathrm{PH}:: \mathrm{PLC}$ VWA-CTM + PH-CTM

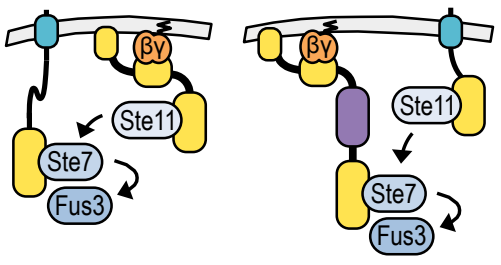

FIGURE 4: Stimulus-mediated regulation of individual Ste5 functions.

(A) Coexpression of Ste5 $\triangle$ VWA and Ste5 $\triangle \mathrm{PH}:: \mathrm{PLC}$ (diagrammed at left) yields trans signaling, and both require intact PM and RING-H2 domains. FUS1-lacZ induction (mean $\pm \mathrm{SD}$; $\mathrm{n}=4$ ) was assayed $\pm \alpha$ factor $\left(5 \mu \mathrm{M}, 2 \mathrm{hr}\right.$.). Strain PPY2032 harbored $\mathrm{HA}_{3}$ - and myc $\mathrm{m}_{13^{-}}$ tagged variants of Ste5 $\triangle$ VWA and Ste5 $\triangle \mathrm{PH}:: \mathrm{PLC}$, respectively.

(B) Cartoon depiction of the signaling scenario in panel $A$.

(C) Combination of a stimulus-regulated partner (Ste5 $\triangle$ VWA or Ste5 $\Delta \mathrm{PH}:: \mathrm{PLC}$ ) with an unregulated, membrane-tethered partner (VWACTM or PH-CTM) yields stimulus-regulated signaling. FUS1-lacZ induction (mean $\pm S D ; n=4$ ) was assayed after induction with galactose $\pm \alpha$ factor $(5 \mu \mathrm{M}, 3 \mathrm{hr})$. Strain: PPY858. Also see Figure S3.

(D) Cartoon depictions of the signaling scenarios in panel $\mathrm{C}$. 
bioRxiv preprint doi: https://doi.org/10.1101/673855; this version posted June 17, 2019. The copyright holder for this preprint (which was not certified by peer review) is the author/funder, who has granted bioRxiv a license to display the preprint in perpetuity. It is made available under aCC-BY-NC 4.0 International license.

Figure 5

A

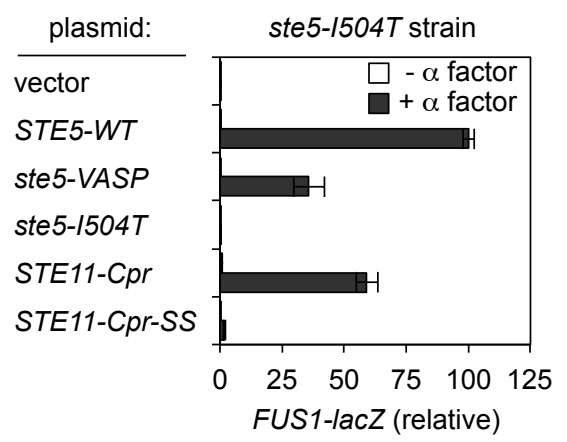

C

Ste20[N]-

Ste11[C]:

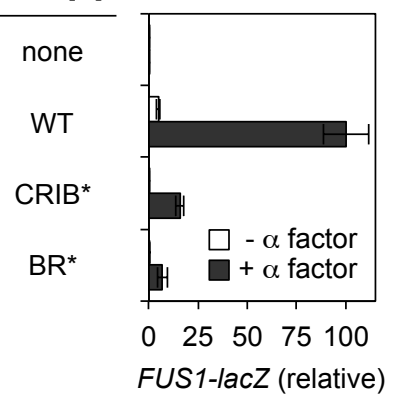

E

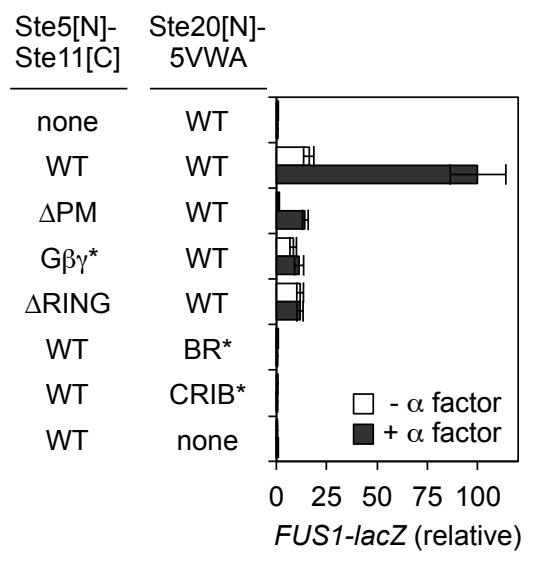

B

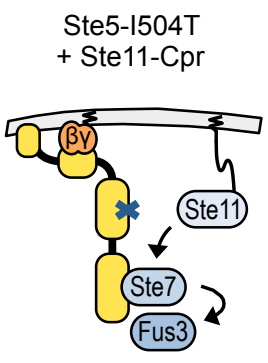

FUS1-lacZ (relative)

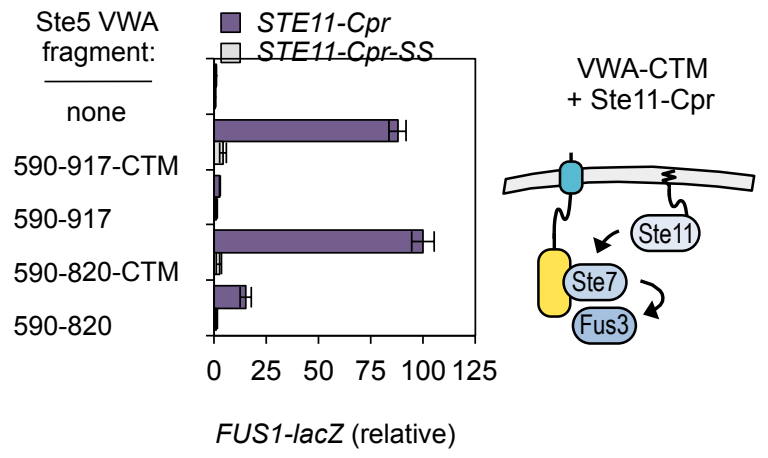

D Ste5-I504T +
Ste20[N]-Ste11[C]
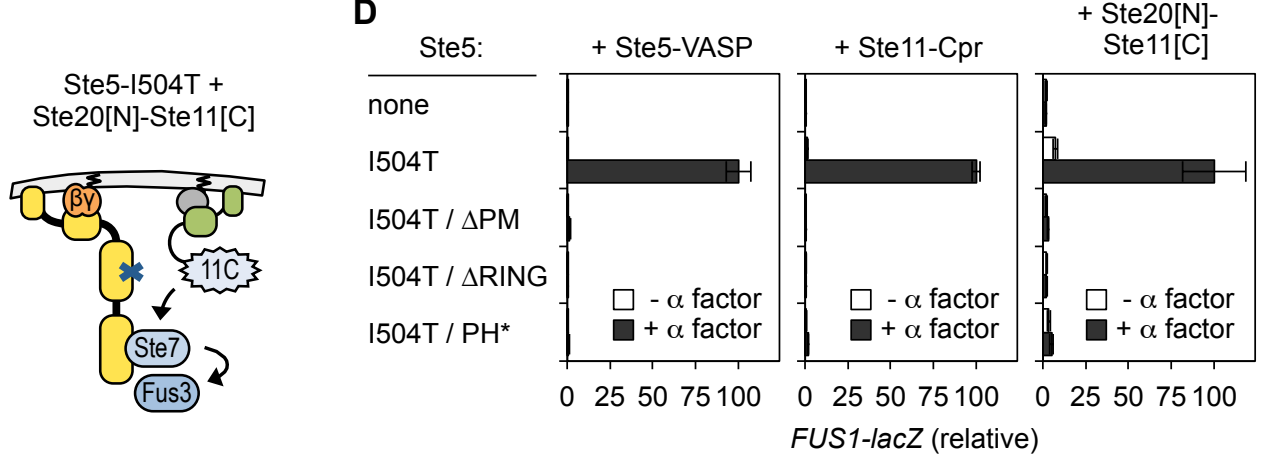

$\mathbf{F}$

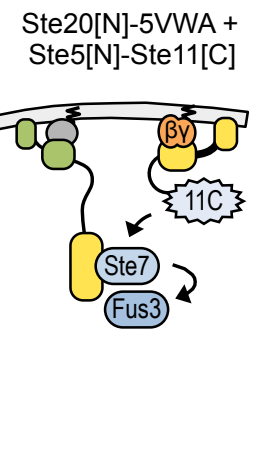

FIGURE 5: Trans signaling by membrane-tethered kinases and alternate recruitment methods.

(A) Complementation of the Ste5-1504T signaling defect by membrane-tethered Ste11 (Ste11-Cpr). FUS1-lacZ induction (mean \pm SD; $\mathrm{n}=$ 4) was assayed in ste5-I504T cells (PPY1968) harboring STE5-myc $c_{13}$ or $P_{G A L 1}$-STE11-Cpr plasmids, after induction with galactose $\pm \alpha$ factor $(5 \mu \mathrm{M}, 3 \mathrm{hr})$.

(B) FUS1-lacZ induction (mean $\pm \mathrm{SD} ; \mathrm{n}=4$ ) in cells (PPY2252) coexpressing the indicated $P_{\text {GAL1 }}$-STE5-CTM and $P_{\text {GAL1 }}$-STE11-Cpr constructs, induced at moderate strength by using $10 \mathrm{nM} \beta$-estradiol (90 min). See also Figure S4A.

(C) Ste5-I504T is complemented by Ste20[N]-Ste11[C], where the active Ste11 kinase domain is localized by the Ste20 N-terminus. FUS1-lacZ (mean $\pm S D ; n=4)$ was assayed $\pm \alpha$ factor $(5 \mu \mathrm{M}, 2 \mathrm{hr})$. CRIB* and BR ${ }^{*}$ denote mutations in Ste20 sequences that bind Cdc42 and plasma membrane, respectively [50, 51]. Strain: PPY2032.

(D) Localization sequences are required for Ste5-I504T to complement Ste5-VASP, Ste11-Cpr, or Ste20[N]-Ste11[C]. FUS1-lacZ induction (mean \pm SD) was assayed $\pm \alpha$ factor $(5 \mu \mathrm{M}, 2 \mathrm{hr} ; \mathrm{n}=4$; PPY2032), or for Ste11-Cpr after induction with galactose $\pm \alpha$ factor (5 $\mu \mathrm{M}, 3 \mathrm{hr} ; \mathrm{n}$ = 3; PPY858). Also see Figure S4A.

(E) FUS1-lacZ induction (mean $\pm \mathrm{SD} ; \mathrm{n}=6$ ) in ste5 $\Delta$ cells (PPY2032) harboring plasmid-borne Ste5[N]-Ste11[C] and Ste20[N]-Ste5[VWA] variants, treated $\pm \alpha$ factor $(5 \mu \mathrm{M}, 2 \mathrm{hr})$.

(F) FUS1-lacZ induction (mean \pm SD; $n=6$ ) in ste5 cells (PPY2032) harboring plasmid-borne Ste5[N]-Ste11[C] and Ste5 4 PH::PLC variants, treated $\pm \alpha$ factor $(5 \mu \mathrm{M}, 2 \mathrm{hr})$. 
bioRxiv preprint doi: https://doi.org/10.1101/673855; this version posted June 17 2019. The copyright holder for this preprint (which was not certified by peer review) is the author/funder, who has granted bioRxiv a license to display the preprint in perpetuity. It is made available under aCC-BY-NC 4.0 International license.

Figure 6

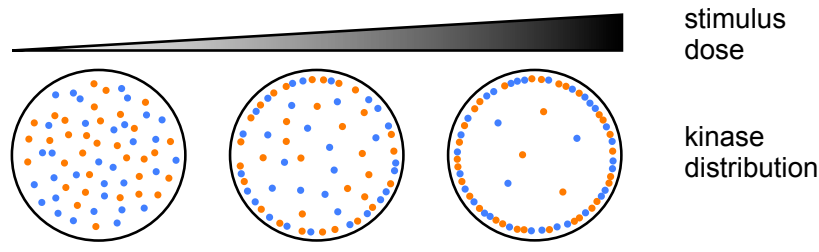

FIGURE 6: General model for concentrating kinases by membrane colocalization.

As the stimulus dose increases, an increasing fraction of molecules is redistributed to a reduced volume of cytoplasm adjacent to the plasma membrane. The increased concentration can favor protein-protein interactions and catalytic reactions among signaling proteins. Results in this study suggest that a scaffold protein can mediate this process by serving as a membrane-recruited binding sink for distinct kinases that signal to each other without being bound to the same scaffold molecule. 